\title{
What is marrow fibrosis after treatment of neuroblastoma?
}

\author{
G E Turner, M M Reid
}

\begin{abstract}
Aims: To test the hypothesis that the abnormal fibrous stroma of bone marrow found after treatment of disseminated neuroblastoma represents traumatic scarring.
\end{abstract}

Methods: Twenty six restaging bone marrow biopsy specimens from 14 children with disseminated neuroblastoma were compared with 37 from 37 children with acute lymphoblastic leukaemia. They were assessed independently by two observers for the presence of abnormal fibrous stroma. All biopsies were performed 23 to 50 days after the initial diagnostic biopsy, and from the same iliac crest. A further nine restaging bone marrow biopsy specimens from previously unbiopsied iliac crests from four children with neuroblastoma were examined and the appearances of the diagnostic bone marrow specimens in those with neuroblastoma recorded. Between diagnostic and restaging biopsies, the children received combination chemotherapy regimens.

Results: In neuroblastoma abnormal stromal material was observed in 17 of 26 rebiopsied iliac crests as opposed to only 9 of 37 of those with acute lymphoblastic leukaemia ( $p<0.005)$. In addition, four of nine restaging biopsy specimens taken from previously unbiopsied crests in patients with neuroblastoma contained abnormal stroma. All 11 children with neuroblastoma whose restaging specimens contained abnormal stroma had similar abnormalities in one or more biopsy specimens at diagnosis.

Conclusions: Abnormal fibrous stromal material in restaging marrow biopsy specimens of previously biopsied iliac crests occurs much more frequently in neuroblastoma than in acute lymphoblastic leukaemia. We suggest that factors other than traumatic scarring are responsible. This is supported by finding similar abnormalities in previously unbiopsied iliac crests and in the diagnostic biopsy specimens of children with neuroblastoma.

(f Clin Pathol 1993;46:61-63)

A recent case report suggested that repeat bone marrow biopsy specimens should not be taken from the previously biopsied iliac crest. ${ }^{1}$ The stages of normal fracture healing may be misinterpreted as myelofibrosis. This observation may be of considerable importance in disseminated neuroblastoma, where biopsy specimens from more than one iliac crest are recommended as part of routine diagnostic staging and assessment of response to treatment. $^{23}$ The marrow appearance of stage 4 neuroblastoma at diagnosis is characterised by an infiltrate of primitive cells with increased reticulin and fibrous stroma ${ }^{4}$ which may persist after chemotherapy in the absence of a detectable malignant infiltrate. ${ }^{5}$

\section{Methods}

All children with disseminated neuroblastoma and acute lymphoblastic leukaemia (ALL) presenting between 1987 and 1991, in whom the biopsied iliac crests were specifically identified, were included in this study. Those with neuroblastoma were aged 1 to 6 years (median 2.5) and with ALL 1 to 14 years (median 4.5) at diagnosis. Twenty six bone marrow biopsy specimens from 14 children with disseminated neuroblastoma and 37 from 37 children with ALL were assessed for the presence of fibrous stroma. All were second-look biopsy specimens taken from the previously biopsied iliac crests. The mean time to second biopsy in ALL was 28 days and in neuroblastoma 43 days. In the interim the children with neuroblastoma were treated with a high dose, rapid schedule regimen $^{6}$ and those with ALL according to the Medical Research Council's UKALL X trial. A further nine restaging biopsy specimens from previously unbiopsied iliac crests from four children with disseminated neuroblastoma were examined. The presence of abnormal fibrous stroma noted in any of the bone marrow biopsy specimens obtained at diagnosis from those with neuroblastoma was also recorded.

The specimens were placed in $10 \%$ neutral buffered formalin, decalcified with Gooding's and Stewart's medium, and embedded in paraffin wax. Sections were stained with haematoxylin and eosin and by Foot's silver reticulin method. Slides were identified by number. We independently reviewed the slides, determining whether sections contained abnormal fibrous stroma. ${ }^{57}$ Any which did so were deemed positive. Of the 72 biopsy specimens examined, concordant results were obtained in $65(90 \%)$. In seven the slides were reviewed jointly before a final decision was made to reach agreement. The numerical code
Correspondence to: Dr M M Reid 
Abnormal fibrous stroma in previously biopsied iliac crests in neuroblastoma and $A L L$

\begin{tabular}{lll}
\hline & Neuroblastoma & ALL \\
\hline Fibrous stoma & $17(65 \% ; 44-83 \%)^{\star}$ & $9(24 \% ; 12-41 \%)$ \\
No abnormal & 9 & 28 \\
stroma & 26 & 37 \\
Total & $\left.\chi^{2}=8.99, \mathrm{p}<0.005\right)$ \\
& \\
\hline
\end{tabular}

$\star(\%$ positive; $95 \%$ confidence limits $)$

\section{was then broken.}

The $\chi^{2}$ test, with Yates's correction, was used to analyse results.

\section{Results}

The results of second-look biopsy specimens from previously biopsied crests are summarised in the table. Fibrous stroma was significantly more likely to be found in those with neuroblastoma than ALL. Four of nine restaging biopsy specimens from previously unbiopsied iliac crests in neuroblastoma were

Figure 1 Low power view of typical second look bone marrow biopsy specimen in disseminated neuroblastoma showing abnormal stroma and architecture

(haematoxylin and eosin).
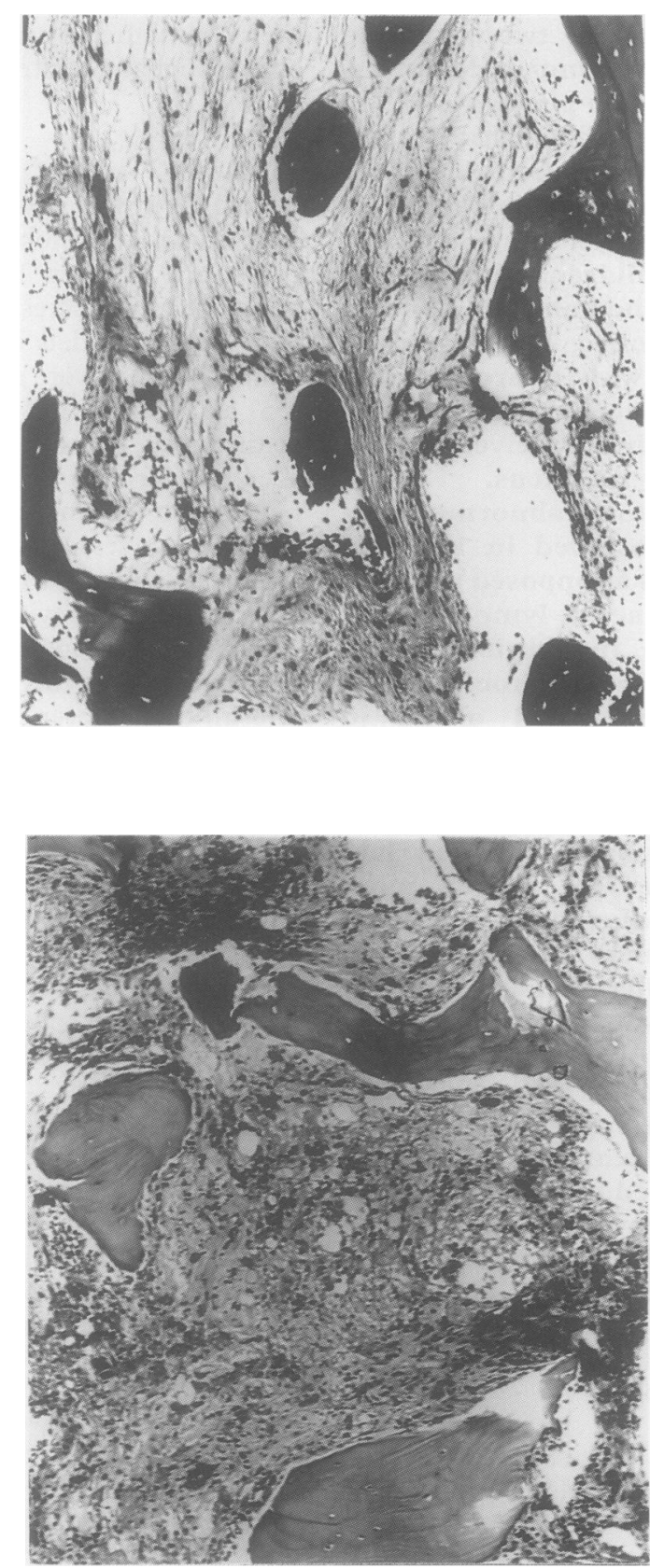

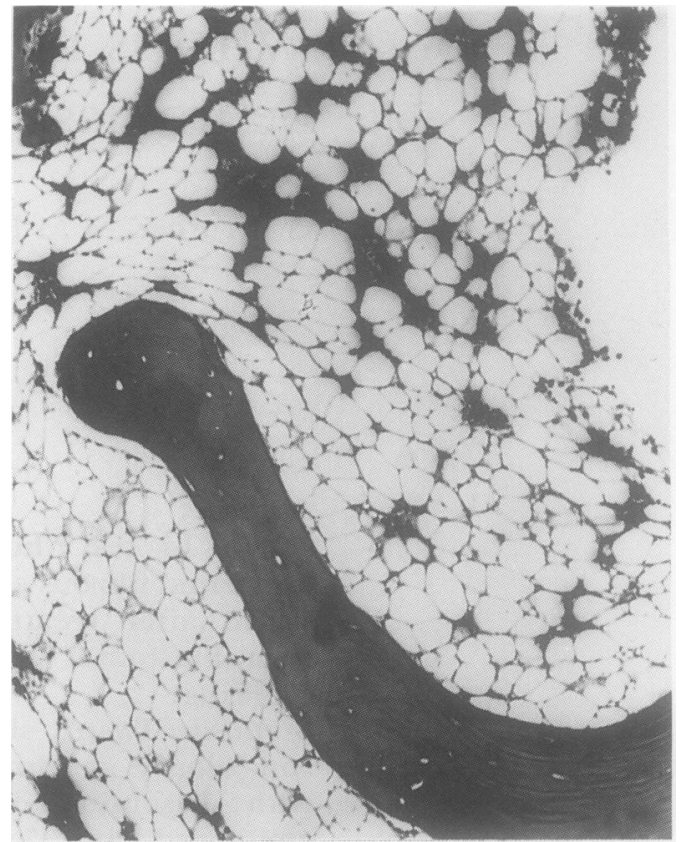

Figure 3 Typical second-look bone marrow biopsy specimen in ALL showing normal architecture (haematoxylin and eosin).

also positive. In neuroblastoma the 21 restaging biopsy specimens containing abnormal fibrous stroma were obtained from 11 children all of whom had similar appearances reported at diagnosis in one or more specimens. Examples of typical restaging marrow biopsy specimens are shown in figs 1 to 3 .

\section{Discussion}

The problems of interpreting the marrow appearances in treated neuroblastoma have been discussed. ${ }^{5}$ Although primitive cells may be hard to find, the marrow often remains abnormal with fibrous stroma and increased reticulin. As restaging biopsy specimens are often taken from previously biopsied iliac crests traumatic scarring could account in part for the appearances of the second biopsy specimens.

While originally only of theoretical concern, the recent description of misinterpretation of post-traumatic scarring as myelofibrosis ${ }^{1}$ prompted a formal investigation of this possibility in children with disseminated neuroblastoma. Certainly one of the patients described in that report ${ }^{1}$ had sustained extensive trauma to the iliac crest; bone marrow harvesting requires multiple punctures, in contrast to a single bone marrow aspirate and biopsy. We thought it unlikely that second-look biopsy specimens would contain any traumatic scarring caused by the original examination.

It is difficult to find appropriate control patients for such a study. In this centre most children with ALL are biopsied both at diagnosis and at the time when remission is confirmed. This archival material does provide a useful, if not ideal, group for comparison: the age range is similar and although the time interval between biopsies is not the same (mean of 28 days for ALL $v 43$ days for 
neuroblastoma) there has been sufficient time for post-traumatic scarring to have developed in those with ALL. ${ }^{8}$ To minimise the effects of our intrinsic bias we conducted this study without knowledge of patients' identities. The attempted "blinding" was only partially successful. On several occasions the histological features were so characteristic that we could correctly identify the disease, although this was not the purpose of the study.

It is conceivable that the histological appearances of restaging biopsy specimens were influenced by the different treatments received by each group. Thus we can not exclude the possibility that the drugs used to treat neuroblastoma were responsible in part for the appearances we have found. Conversely, the treatment of ALL might prevent traumatic scarring of the bone marrow.

Within these limitations this study suggests that traumatic scarring is unlikely to contribute substantially to the marrow appearances after treatment in neuroblastoma. First, abnormal fibrous stroma was significantly more likely to be present in previously biopsied patients with neuroblastoma than ALL. Secondly, it was present in previously unbiopsied iliac crests of some patients with neuroblastoma. Finally, all those with abnormal fibrous stroma at restaging had similar abnormalities at diagnosis.

As already suggested, ${ }^{57}$ the abnormal fibrous stroma which distorts the bone marrow of these children may represent a reaction of normal marrow constituents to occult tumour, be secreted by tumour, or consist of "mature" neuroectodermal tissue. This study does not test these hypotheses, but it does seem more likely that the abnormal stroma is an intrinsic part of the disease than merely post-traumatic artefact.

We thank $\mathrm{Mr} \mathrm{K}$ Brigham for processing the photomicrographs.

1 Salgado C, Feliu E, Blade J, Rozman M, Aguilar JLL, Rozman C. A second bone marrow biopsy as a cause of a false diagnosis of myelofibrosis. $\mathrm{Br} f$ Haematol false diagnosis

2 Franklin IM, Pritchard J. Detection of bone marrow invasion by neuroblastoma is improved by sampling at two sites with both aspirates and trephines. $f$ Clin Pathol 1983;36:1212-8.

3 Brodeur GM, Seeger RC, Barrett A, et al. International criteria for diagnosis, staging and response to treatment in patients with neuroblastoma. $\mathcal{f}$ Clin Oncol 1988; 6:1874-81.

4 Mills AE, Bird AR. Bone marrow changes in neuroblastoma. Pediatr Pathol 1986;5:225-34.

5 Reid MM, Hamilton PJ. Histology of neuroblastoma involving bone marrow: the problem of detecting residual tumour after initiation of chemotheraphy. $\mathrm{Br} \mathcal{F}$ Haematol 1988;69:487-90.

6 Pearson ADJ, Craft AW, Pinkerton CR, Meller ST, Reid MM. High dose rapid schedule chemotherapy for disseminated neuroblastoma. Eur $\mathcal{f}$ Cancer 1992;28A:1654-9.

7 Reid MM, Wallis JP, McGuckin AG, Pearson ADJ, Malcolm AJ. Routine histological compared with immunohistological examination of bone marrow trephine biopsy specimens in disseminated neuroblastoma. $\mathcal{f}$ Clin Pathol 1991;44:483-6.

8 Malcolm AJ. Basics of fracture healing. In: Gregg PJ, Oxford: Blackwell Scientific Publications. (in press). 